\title{
Development of Disc Friction Joining and its Application to Dissimilar Butt Joining of Aluminum and Resin Plates
}

\author{
Toshiya Shibayanagi ${ }^{\mathrm{a},}{ }^{*}$, Norihiro Tajiri $^{\mathrm{b}}$, Shyuhei Hirose ${ }^{\mathrm{c}}$, Satoru Ishihara ${ }^{\mathrm{a}}$ and Masaru Sato ${ }^{\mathrm{d}}$ \\ ${ }^{a}$ Department of Materials Design and Engineering, University of Toyama, 3190 Gofuku, Toyama, 930-8555, Japan \\ ${ }^{b}$ Graduate School of Science and Engineering for education, University of Toyama, Toyama, Japan \\ ${ }^{c}$ Graduate School of University of Toyama, (present: KYB Corporation, Gifu, Japan) \\ ${ }^{d}$ Graduate School of University of Toyama, (present: Toyama Industrial Technology Research and development Center, Toyama, Japan)
}

\begin{abstract}
We have developed a joining process which we named disc friction joining (DFJ). DFJ is a solid state butt joining method that utilizes a rotating disc that is sandwiched and compressed by two plates to be joined. Frictional heat generated at the contact interfaces effectively softens the materials to be plastically deformed by the compression force applied, yielding a joint interface once the disc is withdrawn and the plates make contact. The feasibility of DFJ was examined by joining commercial pure aluminum and polyethylene terephthalate (PET) plates. These materials were successfully joined, and the joint exhibited a tensile fracture stress of $17.1 \mathrm{MPa}$ at room temperature.
\end{abstract}

Key Words: dissimilar joining, rotating disc, aluminum, polyethylene terephthalate, interfaces, tensile strength

\section{INTRODUCTION}

Joining and welding are essential technologies fabricating industrial products. Solid-state joining methods, such as diffusion bonding ${ }^{1)}$, rotary friction welding ${ }^{2)}$, ultrasonic welding ${ }^{3)}$, friction stir welding $(\mathrm{FSW})^{4}$, friction stir spot welding ${ }^{5)}$, and linear friction joining ${ }^{6,7)}$ are widely utilized for materials such as aluminum alloys or dissimilar materials, since they are not easily welded by conventional fusion welding ${ }^{8)}$.

Dissimilar joining is a key for realizing the Sustainable Development Goals (SDG's) $)^{9}$, since energy-conscious engineering such as the production of cutting-edge high-mileage-vehicles, requires "multi-material-structures" composed of light and functional materials.

Welded joints inevitably possess peculiar microstructures near the joint interface that are different from that of the base metal because of the heat and plastic deformation caused by the welding process. This results in discontinuities of the mechanical properties of the joints. In particular, FSW produces a unique microstructure called onion rings ${ }^{4}$. Although FSW is a suitable linear welding technique for metallic plates, it modifies the microstructure of a large portion of the joint. Thus, there is a need to develop a novel joining method to achieve linear welding with minimal effects on the joint microstructure.

We have designed a new welding method based on friction welding which was presented in a previous report on the use of a rotating disc to join bars. However, no attempt was made to join plates ${ }^{10)}$. The present welding method is applicable for the continuous and linear butt joining of plates. Furthermore, it is possible to join metals

\footnotetext{
Received: 2018.10.13 ; accepted: 2019.2.7

*Corresponding author

E-mail address: toshiya@sus.u-toyama.ac.jp
}

to resins, which is difficult to achieve with fusion welding and FSW.

The present study was the first to examine the feasibility of disc friction joining (DFJ) for the dissimilar joining of metals to resins, such as an aluminum plate to a polyethylene terephthalate (PET) resin plate.

\section{EXPERIMENTAL PROCEDURE}

\subsection{Disc Friction Joining}

DFJ comprises two fundamental processes, friction and forging, which are schematically shown in Fig.1 (a). The friction process generates frictional heat between the rotating disc and contacting plates, which are compressed perpendicularly to the disc. The subsequent process is called forging, where the plates meet each other under compression just after passing the periphery of the disc.

The outline of the DFJ process is shown schematically in Figs.1-(b) - (f). Two plates are firmly fixed on the sliding apparatus of the joining machine (b). Subsequently, a disc sandwiched by these plates begins to rotate by a servo-motor with a fixed rotation speed (c). The disc is simultaneously compressed by the plates via a gas-pressure module (d). The plate materials are partially softened near the contact interface due to frictional heating. After a few seconds of frictional heating, the rotating disc is withdrawn from the plates (e), and a joint between the plates forms (f). The welding direction is the withdrawing direction of the disc.

\subsection{Joining conditions and evaluation of joints}

The materials used were commercial pure aluminum A1070-H24 and PET resin plates. The specimens for the joining tests had $2 \mathrm{~mm}$ thicknesses, $30 \mathrm{~mm}$ lengths, and $10 \mathrm{~mm}$ widths. A disc made of SKH51 steel with a $140 \mathrm{~mm}$ diameter and $0.5 \mathrm{~mm}$ thickness was used with rotation 


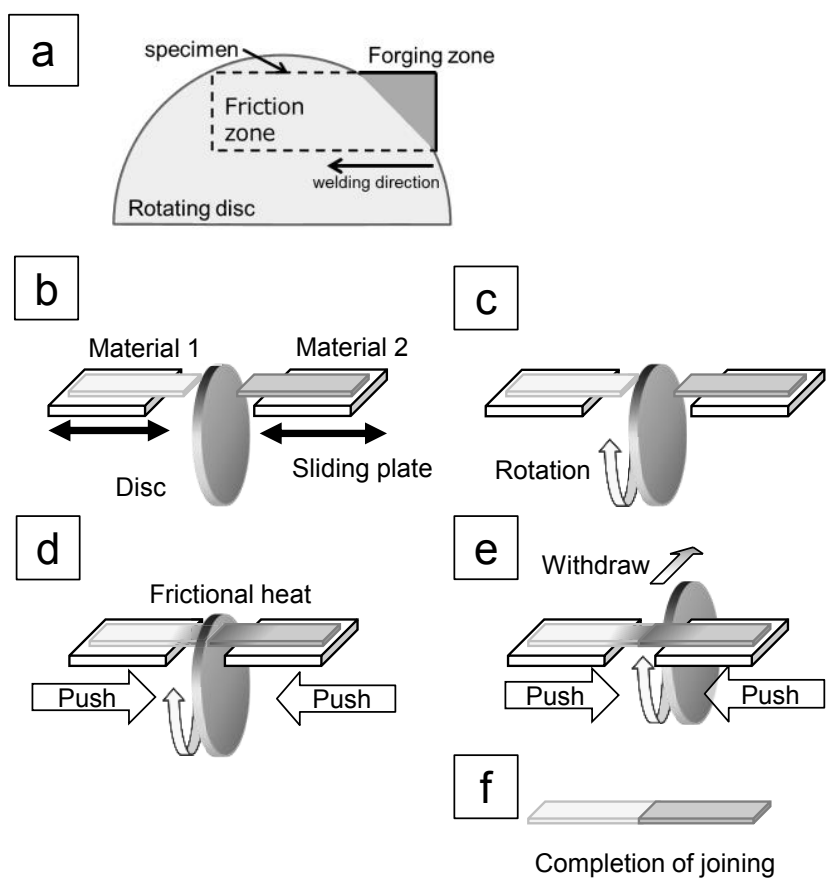

Fig.1 Schematic illustration of the disc friction joining process.

speeds up to $2000 \mathrm{rpm}$. The gas pressure to provide a compression force of $23 \mathrm{~N}$ on the joint interface was applied for the entire joining.

After $2 \mathrm{~s}$ of frictional heating, the rotating disc was quickly withdrawn to allow the plates to contact under pressure. K-type thermocouples were spot welded to record the temperature history at points $1 \mathrm{~mm}$ from the joint interface during joining. The temperatures on both sides of the plates were measured.

An optical microscope was used to examine defects and evaluate the microstructures of the joints after mechanical polishing of the cross-sections with emery papers and diamond paste. Tensile tests of the joints were carried out at ambient temperature with an initial strain rate of $5.6 \mathrm{x}$ $10^{-4} \mathrm{~s}^{-1}$ after removing the burr. The tensile specimens were prepared after removing the burr followed by polishing to yield flat surfaces, and the dimension was $8 \mathrm{~mm} \times 55 \mathrm{~mm} \times 1.6 \mathrm{~mm}$. The actual gauge length was $30 \mathrm{~mm}$. The fractured surfaces of the tensile tested specimens were observed with a low-vacuum scanning electron microscope, (Hitatchi Miniscope TM3030).

\section{RESULT AND DISCUSSION}

The top and bottom views and cross-section of an A1070/PET joint produced by DFJ are shown in Fig.2. The top view (a) of the joint, in which 1070 aluminum is on the right side, clearly shows that a burr of the resin formed along the direction of motion of the rotating disc, i.e. the welding direction as indicated by the arrow. The bottom view (b) of the same joint also shows the burr. Based on this observation, the joint formation is mainly attributed to the deformation or flow of resin at the contact surfaces during the forging process.

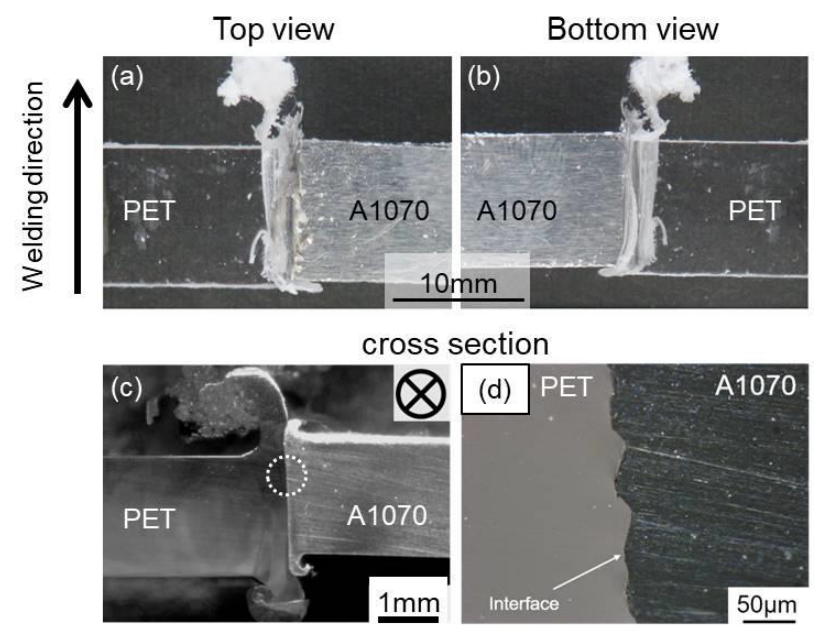

Fig.2 Top and bottom views and cross-section of A1070/PET joint produced by DFJ,:

(a) top view, (b) bottom view, (c) macroscopic view of cross section, and (d) magnified image of the white circle in (c), showing an uneven interface.

No significant defects such as tunnels or grooves, were observed, in the macroscopic and magnified images shown in Figs.2 (c) and, (d). The mark on the right top side in Fig. 2 (c) indicates the welding direction perpendicularly towards the other side. Thus the DFJ produced a high quality joint with no defects throughout the thickness. The magnified view of the interfacial region shows a zig-zag shape of the interface, i.e., asperities with sizes of several tens of micrometers on the joint interface. This implies that joining is due to an anchoring effect caused by the resin being injected into the pits on the aluminum side, while other mechanisms, such as chemical bonding, should also be considered to fully understand the joining mechanisms of DFJ.

Figure 3 shows temperature histories of the aluminum and the resin, which were measured $1 \mathrm{~mm}$ from the disc in both materials. On the aluminum side, the temperature increased monotonically as the friction heating occurred and reached a maximum temperature of $576.6 \mathrm{~K}$ after $2 \mathrm{~s}$. As commercial grade aluminum is softened above around

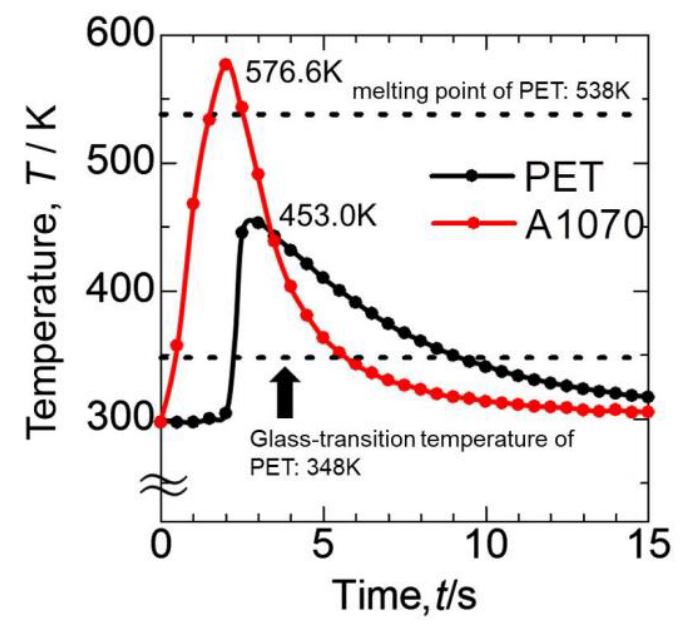

Fig.3 Temperature histories during DFJ $1 \mathrm{~mm}$ from the disc surface in both materials. 
(a)

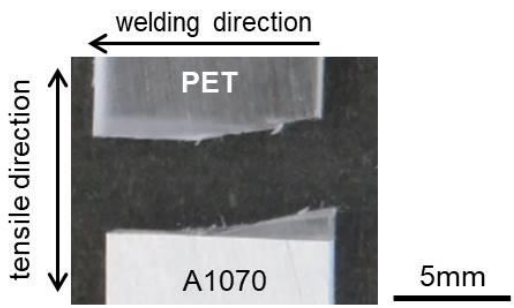

(b) welding direction
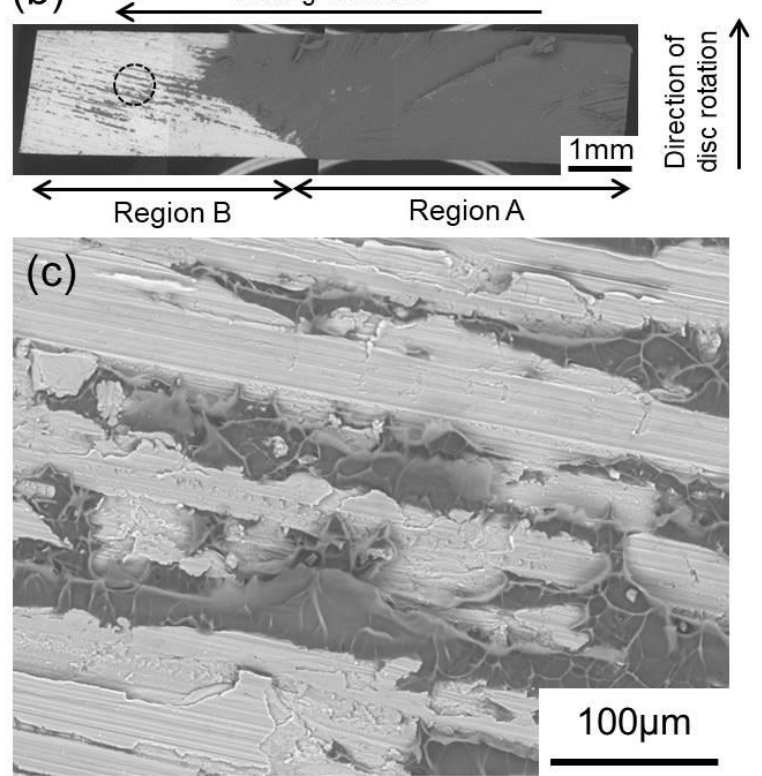

Fig.4 Analysis of fractured surface of the joint tensile-tested at ambient temperature. (a) Macroscopic appearance of the fractured joint showing resin remaining on the aluminum side, (b) SEM image of the fractured surface on the aluminum side, showing two distinct regions with different contrast, where the aluminum is observed as brighter contrast, (c) magnified SEM image from the black dotted circle in (b), showing resin in the grooves on the aluminum plate.

$473 \mathrm{~K}^{11)}$, the aluminum plate was softened and plastically deformed only near the disc during frictional heating. This is consistent with the deformation observed in Fig.2. On the PET side, the temperature also increased and reached a maximum temperature of $453.0 \mathrm{~K}$, which is above its glass transition temperature of $348 \mathrm{~K}$. Thus, the resin was soft enough to flow and be injected into the small asperities, such as hollow regions or pits, on the contact cross-section of the aluminum plate. Therefore, the friction between the disc and plates generated sufficient heat for the resin to join with the aluminum plate through a mechanical bond in the surface asperities.

The fracture strength of the dissimilar joint was 17.0MPa, which is low compared to PET joints, which range from 48 to $73 \mathrm{MPa}$. These values for PET were measured by tensile tests for PET plates in the present study. Observation of fractured surfaces was conducted for the tensile-tested joint. Figure 4 shows the macroscopic appearance (a), the fractured surface on the aluminum side (b), and its magnified view (c). The fracture occurred partially in the resin as seen in (a), suggesting a strong interface was achieved. In other words, two fracture paths are clearly observed in (a) and (b), called regions A and B.
Region A proves that the fracture occurred in the resin, and this was also confirmed by scanning electron microscopy. Region B shows a fragment of resin with dark contrast sticking to the grooves on the aluminum surface, indicating that the joint is created by an anchoring effect between the two materials.

\section{CONCLUSIONS}

In the present study, the feasibility of DFJ for dissimilar joining of commercial pure aluminum to PET as subject materials was examined. Friction between the rotating disc and these materials yielded joints with no significant defects, as expected. This novel joining technique could be applied to the joining of thin plates, pipes, or on-site joining of reinforcements at construction sites.

The joining was achieved by the so-called anchor effect, but further investigation is required in terms of chemical bonding at the joint interface, tribology, and especially control of the friction coefficients on each side of the disc.

\section{Acknowledgements}

The present work was partly supported by JSPS KAKENHI (Grant number JP18K04773), and the Amada Foundation (Grant number AF-2017022).

\section{Reference}

1) H. Kokawa, T. Suzuki and T. Kuwana: Effect of Initial Microstructure of Intermediate Material on Superplastic Diffusion Bonding of Duplex Stainless Steel, ISIJ International, 35-10 (1995), 1291-1297.

2) W. Li, A. Vairis, M. Preuss, Tiejun Ma: Linear and rotary friction welding review, J. International Materials Review, 61-2 (2016), 71-100.

3) H. Ji, Y. Qiao, M. Li: Rapid Formation of Intermetallic Joints through Ultrasonic-Assisted Die Bonding with $\mathrm{Sn}-0.7 \mathrm{Cu}$ Solder for High Temperature Packaging Application, Scripta Mater., 110-1 (2016), 19-23.

4) R. S. Mishra, Z. Y. Ma: Friction Stir Welding and Processing, Mater. Sci. Eng. R, 50-1-2 (2005) 1-78.

5) A. Ikuta, Y. H. Yin, T. H. North: Influence of Tool Thread on Mechanical Properties of Dissimilar Al Alloy Friction Stir Spot Welds, Sci. Tech. Welding \& Joining, 17-8 (2012), 622-629.

6) S. Bray, Rolls-Royce plc, GB Patent Application No. GB0611926.7, June (2006).

7) I. Bhamji, M. Preuss, P. L. Threadgill, A. C. Addison: Solid State Joining of Metals by Linear Friction Welding, Mater. Sci. Tech., 27-1 (2011), 2-12.

8) Y. Zhang, J. Huang, Z. Cheng, Z. Ye, H. Chi, L. Peng, S. Chen: Study on MIG-TIG double-sided arc welding-brazing of aluminum and stainless steel, Materials Letters, 172(2016)146-148.

9) UN General Assembly, Agenda items 15 and 116, 21 Oct., 2015.

10) T. Higashi, R. Tsujino, K. Matsuura, and S. Kikuchi: Development of square cross sectional aluminum alloy joint by new friction welding method, J. of Light Metal Welding, 52-10 (2014), 384- 390. (in Japanese)

11) S. Yamamoto, M. Mizuno, A. Kirihata: Behaviour of $\mathrm{Fe}$ Atoms and Age-Softening in Al-Fe Alloy, J. Japan Inst. Metals, 56-7 (1992), 757-763. (in Japanese) 